\title{
EFEITO DO TRATAMENTO HIDROTÉRMICO ASSOCIADO AO ETANOL NA QUALIDADE DE MANGA TOMMY ATKINS
}

\section{SILVANA BELÉM DE OLIVEIRA VILAR ${ }^{1}$, MARIA FERNANDA PONTES PENTEADO MORETZSOHN DE CASTRO ${ }^{2}$, JOSÉ MARIA MONTEIRO SIGRIST ${ }^{2}$, ANA JÚLIA BRITO ARAÚJO ${ }^{3}$, FLÁVIO LUÍS SCHMIDT ${ }^{1}$}

\footnotetext{
1 Universidade Estadual de Campinas, UNICAMP, Faculdade de Engenharia de Alimentos, Campinas/SP, Brasil, silvanatecal@gmail.com, schmidt.unicamp@gmail.com, ${ }^{2}$ Instituto de Tecnologia de Alimentos, ITAL, Grupo de Engenharia e Pós-Colheita, Campinas/SP, Brasil, fernanda@ital.sp.gov.br; jmms@ital.sp.gov.br, ${ }^{3}$ Instituto Federal do Sertão Pernambucano, Petrolina/PE, Brasil, a.juliaaraujo@gmail.com
}

RESUMO: A manga é uma fruta de grande expressão nas exportações brasileiras, porém, como barreira fitossanitária alguns países importadores exigem que as mesmas recebam um tratamento hidrotérmico para o controle de moscas-das-frutas. Alternativas do uso do etanol no controle de microrganismos e fitopatógenos em vegetais têm sido estudadas. Este trabalho avaliou a influência do tratamento hidrotérmico e sua associação com $1 \%$ de etanol na qualidade de mangas cv. Tommy Atkins armazenadas a $10^{\circ} \mathrm{C} / 90 \% \mathrm{UR}$. Foram realizados experimentos com 200 frutos em estágio de maturação 2, comparando-se o tratamento controle (mangas lavadas, secas e armazenadas), o tratamento hidrotérmico sem etanol e o tratamento combinando o banho hidrotérmico com $1 \%$ de etanol. Concluiu-se que os parâmetros de qualidade das mangas não foram afetados pelo banho hidrotérmico nem pela adição de etanol. O etanol associado ao banho térmico pode ser uma ferramenta no controle de patógenos em mangas, sem afetar a qualidade durante o armazenamento refrigerado nas condições desse estudo.

PALAVRAS-CHAVE: Mangífera índica, álcool, pós-colheita.

\section{EFFECT OF HYDROTHERMALTREATMENT ASSOCIATE TO ETHANOL ON THE QUALITY MANGO 'TOMMY ATKINS'}

\begin{abstract}
Mango is a fruit of great expression in Brazilian exports; however, as phytosanitary limitation some importing countries require that mango receives hydrothermal treatment for the control of the fruit fly. The alternative use of ethanol in the control of microorganisms and pathogens in plants have also been studied. This study evaluated the effect of hydrothermal treatment and its association with $1 \%$ ethanol on the quality of mango cv. Tommy Atkins stored at $10^{\circ} \mathrm{C} / 90 \%$ RH. Experiments were performed with 200 fruit in maturation stage 2, comparing the control treatment (mango washed, dried, and stored), the hydrothermal treatment without ethanol and combining the hydrothermal treatment with $1 \%$ ethanol. It was concluded that the mango quality parameters were not affected by hydrothermal bath or addition of ethanol. Ethanol associated with the hydrothermal treatment can be a practice to control pathogens in mango, without affecting the quality during storage.
\end{abstract}

KEY WORDS: Mangífera índica, alcohol, post-harvest. 


\section{INTRODUÇÃO}

O Brasil é o terceiro maior produtor mundial de frutas, com uma colheita que excede 40 milhões de toneladas anuais. Estudos realizados por Santos et al. (2013) indicam que a manga está entre as principais frutas de exportação no país, em 2013, esta cadeia produtiva comemorou o recorde de receita nos negócios com o exterior: US\$ 147,5 milhões, sendo que em volume, foram embarcadas 122 mil toneladas da fruta. De acordo com Lopes et al. (2008), as exigências de países importadores de frutos in natura têm sido crescentes em relação à qualidade do produto, especialmente quanto à presença de resíduos de agrotóxicos.

Frutas que são destinadas ao mercado de exportação devem passar através de tratamentos pós-colheita que variam de acordo com as exigências do país importador. $\mathrm{O}$ uso do tratamento hidrotérmico tem sido exigido para evitar problemas com mosca-das-frutas, porém, sua boa eficiência no controle de fungos e a proibição do uso de fungicidas na póscolheita pelo Food and Drug Administration, em 1986 (USDA-APHIS, 2002), fez com que ele fosse estabelecido como método quarentenário para mangas exportadas para a Europa, Estados Unidos e Japão. Ainda existe pouca ciência sobre os efeitos bioquímicos e microbiológicos que este tratamento pode causar aos frutos. Este estudo associou etanol ao banho térmico, pois existem indícios que o etanol possui uma potente atividade antimicrobiana, incluindo efeitos positivos do etanol no controle de doenças pós-colheita em diversas culturas (PESIS, 2005).

Em estudos com uvas naturalmente infectadas com esporos de Botrytis cinérea, Karabulut et al. (2004) constataram que a imersão dos frutos por 30 segundos em $30 \%$ de etanol a $24{ }^{\circ} \mathrm{C}$, reduziu em $50 \%$ a incidência de doenças, após 35 dias de armazenamento a 1 ${ }^{\circ} \mathrm{C}$, observando-se ainda que a combinação do banho térmico a $50^{\circ} \mathrm{C} / 3 \mathrm{~min} \mathrm{com} 10 \%$ de etanol reduziu de forma significativa a incidência do fungo em uvas artificialmente inoculadas. Lichter et al. (2002) também ao estudarem uvas, relataram que a imersão de seus cachos em 33, 40 ou $50 \%$ de etanol por 30 minutos, resultou na redução de doenças póscolheita, mostrando-se tão ou mais efetivo que o dióxido de enxofre $\left(\mathrm{SO}_{2}\right)$, além de não prejudicar a aparência dos frutos.

Para patógenos veiculados por frutas, estudos realizados por Rezende et al (2009) indicaram que a imersão de goiabas inoculadas com Salmonella Enteritidis e E. coli em etanol por 2 minutos e em banho hidrotérmico $\left(50^{\circ} \mathrm{C} / 10 \mathrm{~min}\right)$ reduziram em média $53 \%$ da população daqueles microrganismos.

O objetivo desse trabalho foi avaliar o efeito do tratamento hidrotérmico e do tratamento hidrotérmico associado a $1 \%$ de etanol na qualidade pós-colheita de mangas cv. Tommy Atkins.

\section{MATERIAL E MÉTODOS}

Foram utilizadas 200 mangas cv. Tommy Atkins provenientes de Petrolina, Pernambuco no estágio de maturação 2, conforme escalas propostas por Protrade (1992), considerada como referência para a colheita dessa cultivar. Foram realizados os seguintes tratamentos: Controle absoluto (manga apenas lavada, seca e armazenada - T1), controle (manga submetida ao banho hidrotérmico sem etanol - T2) e banho hidrotérmico com 1\% de etanol (T3).

Os experimentos foram conduzidos no Grupo de Engenharia Pós-colheita (GEPC) do Instituto de tecnologia de alimentos (ITAL, Campinas-SP). O tratamento térmico consistiu na imersão dos frutos em um banho de escala semi-piloto (capacidade para 250 litros de água) a 
$46,1^{\circ} \mathrm{C} / 75 \mathrm{~min}$. Após tratamento hidrotérmico realizou-se o arrefecimento num outro banho a $21,1^{\circ} \mathrm{C} / 30$ minutos, sempre utilizando a água tratada do sistema municipal de Campinas - SP. A temperatura da água foi medida com termômetro de mercúrio graduado com capacidade de até $100{ }^{\circ} \mathrm{C}$. Quando o tratamento hidrotérmico foi realizado com etanol, seu efeito sobre as características físico-químicas e sensoriais da manga foram avaliados. Depois dos tratamentos os frutos foram secos com papel toalha e armazenados a $10^{\circ} \mathrm{C} / 90 \%$ UR durante 14 dias com transferência para $25^{\circ} \mathrm{C} / 75 \% \mathrm{UR}$ por um tempo adicional de 6 dias. Ao longo de todo o armazenamento as mangas foram analisadas a cada 2 ou 3 dias.

Para as análises físico-químicas foram utilizados 10 frutos por determinação. A firmeza da polpa foi determinada pela leitura de dois pontos na região equatorial dos frutos após a retirada da casca, através do texturômetro TA-XT2i (Stable Microsystems) equipado com um cilindro de $35 \mathrm{~mm}$ de diâmetro; O conteúdo de sólidos solúveis (SS) em um refratômetro portátil Atago (Atago Co. Ltd., Tóquio, Japão) com uma escala de 0 a $32^{\circ}$ Brix. $\mathrm{O}$ valor do $\mathrm{pH}$ foi obtido pelo método potenciométrico, com um medidor de $\mathrm{pH}$ Tecnopon, modelo MPA 210/mPA - 210p; a acidez titulável (g de equivalente ácido cítrico por $100 \mathrm{~g}^{-1}$ ) foi determinada por titulação com $\mathrm{NaOH} 0,1 \mathrm{~N}$ até pH 8,1 (AOAC, 1997); a cor da casca e da polpa de cada fruto, pelo Sistema CIE L*C* $\mathrm{H}^{*}$ em um colorímetro Minolta modelo CR-300 (Minolta Camera Co, Japão).

$\mathrm{Na}$ análise da aparência dos frutos, utilizou-se o método de comparação múltipla (MORAES, 1988). Os testes foram aplicados no $7^{\circ}, 14^{\circ}, 17^{\circ}$ e $19^{\circ}$ dias de armazenamento. A avaliação foi realizada com 15 observadores não treinados de ambos os sexos. A iluminação foi feita utilizando-se luz branca. As mangas foram servidas simultaneamente, em bandejas brancas, codificados com números aleatórios de três dígitos. As posições foram casualizadas entre os observadores. Os avaliadores receberam uma amostra Controle (fruto submetido ao tratamento hidrotérmico sem etanol) e as demais codificadas, sendo uma destas o próprio controle e preencheram uma ficha de avaliação sensorial para verificar o efeito do tratamento hidrotérmico com e sem etanol na aparência dos frutos. Utilizou-se uma escala que compreendia nove pontos, sendo que 0 (zero) $=$ nenhuma diferença e 9 (nove) $=$ extremamente diferente.

Os dados foram submetidos à análise de variância e as médias comparadas pelo teste de Tukey (determinações físico-químicas) e teste de Dunnet (aparência dos frutos) em delineamento inteiramente casualizado. A discussão dos resultados foi efetuada a $5 \%$ de significância. Utilizou-se o software SAS.

\section{RESULTADOS E DISCUSSÃO}

Observou-se um aumento no teor de sólidos solúveis em todos os tratamentos (Tabela 1). Houve diferença significativa entre os tratamentos no $7^{\circ}$ e $14^{\circ}$ dia, quando se observaram, nesses períodos, maiores valores de sólidos solúveis para o tratamento alcoólico em relação aos demais tratamentos, porém, com o passar do tempo essa diferença não foi estatisticamente significativa. Como a manga é um fruto climatérico, esse aumento no teor de sólidos solúveis é esperado, pois durante o processo de amadurecimento de frutos climatéricos, pode-se verificar várias alterações, como na firmeza da polpa, nos teores de açúcares, na coloração da casca e da polpa e na composição e teor de ácidos orgânicos (CHITARRA; CHITARRA, 2005).

Ocorreu diminuição da acidez das mangas durante o armazenamento (Tabela 2). A acidez no $20^{\circ}$ dia foi menor para o tratamento 1 , essa diferença foi estatisticamente significativa e pode indicar que as mangas que não sofreram tratamento hidrotérmico 
apresentavam-se mais maduras que as demais. Esse resultado também é coerente, uma vez que os ácidos orgânicos usualmente declinam em decorrência de sua utilização como substrato na respiração ou da sua transformação em açúcares. Essas transformações têm papel importante nas características de sabor e aroma, uma vez que alguns compostos são voláteis (CHITARRA; CHITARRA, 2005; SILVA et al., 2011). Os resultados encontrados nesse trabalho podem indicar que as mangas sem tratamento térmico apresentaram-se fisiologicamente mais ativas, comportamento este que pode indicar que a atividade respiratória foi retardada pelo tratamento hidrotérmico, estando de acordo com os resultados observados com mamões por Paull e Chen (1989).

Tabela 1. Comparação dos valores médios dos sólidos solúveis ( ${ }^{\circ}$ Brix) das mangas armazenadas $10^{\circ} \mathrm{C}$.

\begin{tabular}{cccccc}
\hline Etanol & 1 Dia & \multicolumn{1}{c}{7 Dias } & 14 Dias & 18 Dias & 20 Dias \\
\hline Cabs & $8,35 \pm 0,54 \mathrm{a}$ & $10,82 \pm 0,72 \mathrm{a}$ & $12,35 \pm 0,74 \mathrm{a}$ & $10,87 \pm 0,54 \mathrm{a}$ & $10,53 \pm 0,40 \mathrm{a}$ \\
Controle & $8,35 \pm 0,54 \mathrm{a}$ & $10,98 \pm 0,35 \mathrm{a}$ & $11,8 \pm 0,57 \mathrm{ab}$ & $11,43 \pm 0,90 \mathrm{a}$ & $11,05 \pm 0,31 \mathrm{a}$ \\
$1 \%$ de etanol & $8,35 \pm 0,54 \mathrm{a}$ & $10,03 \pm 0,38 \mathrm{~b}$ & $11,2 \pm 0,59 \mathrm{~b}$ & $11,73 \pm 0,74 \mathrm{a}$ & $11,00 \pm 0,38 \mathrm{a}$ \\
\hline
\end{tabular}

Letras iguais na mesma Coluna não diferem significativamente entre si a $\mathrm{p} \leq 0,05$ (Teste de Tukey).

Houve aumento do $\mathrm{pH}$ durante o tempo de armazenamento (Tabela 3), porém, pode-se afirmar que estatisticamente não houve diferença significativa importantes entre os tratamentos. Como o ácido cítrico é um ácido orgânico relativamente fraco, as alterações na acidez não causaram grande impacto no $\mathrm{pH}$.

Tabela 2. Comparação dos valores médios da acidez total titulável (\%) das mangas armazenadas $10^{\circ} \mathrm{C}$.

\begin{tabular}{cccccc}
\hline Etanol & 1 Dia & 7 Dias & 14 Dias & 18 Dias & \multicolumn{2}{c}{20 Dias } \\
\hline Cabs & $1,07 \pm 0,10 \mathrm{a}$ & $1,00 \pm 0,10 \mathrm{a}$ & $0,76 \pm 0,05 \mathrm{~b}$ & $0,28 \pm 0,10 \mathrm{a}$ & $0,086 \pm 0,09 \mathrm{~b}$ \\
Controle & $1,07 \pm 0,10 \mathrm{a}$ & $0,95 \pm 0,10 \mathrm{a}$ & $0,71 \pm 0,06 \mathrm{~b}$ & $0,37 \pm 0,07 \mathrm{a}$ & $0,15 \pm 0,06 \mathrm{a}$ \\
$1 \%$ de etanol & $1,07 \pm 0,10 \mathrm{a}$ & $1,07 \pm 0,13 \mathrm{a}$ & $0,97 \pm 0,09 \mathrm{a}$ & $0,30 \pm 0,06 \mathrm{a}$ & $0,15 \pm 0,04 \mathrm{a}$ \\
\hline
\end{tabular}

Letras iguais na mesma Coluna não diferem significativamente entre si a $\mathrm{p} \leq 0,05$ (Teste de Tukey).

A firmeza, pelo que se esperava com o amadurecimento dos frutos, diminuiu com o tempo de armazenamento. Observa-se na Tabela 4 que esta diminuição foi mais rápida no Controle Absoluto (T1). Laurie (1998) afirma que o tratamento térmico tende a diminuir a taxa de amaciamento, uma vez que altas temperaturas inibem a ação de enzimas da parede celular, como a poligalacturonase, corroborando com os resultados aqui apresentados.

Tabela 3. Comparação dos valores médios do $\mathrm{pH}$ das mangas armazenadas $10^{\circ} \mathrm{C}$.

\begin{tabular}{cccccc}
\hline Etanol & 1 Dia & 7 Dias & 14 Dias & \multicolumn{1}{c}{18 Dias } & 20 Dias \\
\hline Cabs & $3,52 \pm 0,07$ a & $3,53 \pm 0,05$ a & $3,60 \pm 0,03$ a & $4,24 \pm 0,22$ ab & $4,88 \pm 0,03$ a \\
Controle & $3,52 \pm 0,07$ a & $3,57 \pm 0,04$ a & $3,68 \pm 0,04$ a & $4,08 \pm 0,12$ b & $4,75 \pm 0,34$ a \\
$1 \%$ de etanol & $3,52 \pm 0,07$ a & $3,55 \pm 0,08$ a & $3,65 \pm 0,08$ a & $4,40 \pm 0,12$ a & $4,83 \pm 0,15$ a \\
\hline
\end{tabular}

Letras iguais na mesma Coluna não diferem significativamente entre si a $\mathrm{p} \leq 0,05$ (Teste de Tukey).

Verificou-se que houve aumento da luminosidade da casca da manga no decorrer do armazenamento (Tabela 5). Constatou-se que as mangas que sofreram tratamento hidrotérmico apresentaram-se mais luminosas. Houve diferenças estatísticas significativas em quase todos os dias analisados, onde $\mathrm{T} 1$ diferiu dos demais tratamentos realizados. A pureza da cor (croma) aumentou com o tempo, indicando o amadurecimento da manga (Tabela 5). Houve diferença significativa entre os tratamentos, sendo que o aumento do croma foi maior nas mangas que não sofreram tratamento hidrotérmico. Em estudos com manga Palmer, 
Santos et al. (2010) observaram que os frutos tratados hidrotermicamente apresentaram intensidade respiratória menor quando comparados com os frutos que não foram submetidos ao tratamento hidrotérmico, durante os primeiros sete dias de armazenamento a $23 \pm 2{ }^{\circ} \mathrm{C}$, $65 \pm 5 \%$ UR, concordando com os resultados do presente estudo.

Tabela 4. Comparação dos valores médios da firmeza $(\mathrm{N})$ das mangas armazenadas $10^{\circ} \mathrm{C}$.

\begin{tabular}{cccccc}
\hline Etanol & 1 Dia & 7 Dias & 14 Dias & 18 Dias & 20 Dias \\
\hline Cabs & $118,49 \pm 10,99$ a & $48,11 \pm 20,85$ b & $20,68 \pm 8,87$ b & $7,98 \pm 1,99 \mathrm{~b}$ & $3,66 \pm 0,26 \mathrm{~b}$ \\
Controle & $118,49 \pm 10,99$ a & $54,52 \pm 15,11$ b & $42,47 \pm 8,47$ a & $12,91 \pm 3,09$ a & $3,88 \pm 0,31 \mathrm{~b}$ \\
$1 \%$ de etanol & $118,49 \pm 10,99 \mathrm{a}$ & $86,26 \pm 20,65$ a & $46,75 \pm 6,76$ a & $12,13 \pm 2,43$ a & $4,74 \pm 0,53 \mathrm{a}$ \\
\hline \multicolumn{7}{l}{ Letras iguais na mesma Coluna não diferem significativamente entre si a $\mathrm{p} \leq 0,05$ (Teste de Tukey). }
\end{tabular}

Os valores médios do ângulo de cor da casca diminuíram com o tempo de armazenamento (Tabela 5), indicando a passagem da cor verde para a coloração laranja (valores entre 0 e $90^{\circ}$ ).

O tratamento hidrotérmico pode ter contribuído para retardar as mudanças relativas à coloração da casca da manga, características do processo de maturação, visto que estas estavam com a coloração mais escura ( $\mathrm{L}^{*}$ menor), com tons mais acinzentados ( $\mathrm{C}^{*}$ menor) e com menos tons vermelhos $\left(\mathrm{H}^{*}\right.$ mais próximo de $90^{\circ}$ ) comparadas às mangas que não sofreram tratamento hidrotérmico.

Tabela 5. Comparação dos valores médios da cor da casca das mangas armazenadas $10^{\circ} \mathrm{C}$.

\begin{tabular}{ccccccc}
\hline Variável & Etanol & 1 Dia & 7 Dias & 14 Dias & 18 Dias & 20 Dias \\
\hline \multirow{4}{*}{$\mathrm{L}^{*}$} & Cabs & $51,38 \pm 0,94 \mathrm{a}$ & $54,29 \pm 0,44 \mathrm{a}$ & $57,70 \pm 1,85 \mathrm{a}$ & $58,24 \pm 1,46 \mathrm{a}$ & $62,43 \pm 2,20 \mathrm{a}$ \\
& Controle & $51,38 \pm 0,94 \mathrm{a}$ & $54,04 \pm 1,39 \mathrm{a}$ & $55,47 \pm 1,99 \mathrm{ab}$ & $57,43 \pm 2,5 \mathrm{ab}$ & $56,48 \pm 2,07 \mathrm{~b}$ \\
& $1 \%$ de etanol & $51,38 \pm 0,94 \mathrm{a}$ & $51,90 \pm 0,92 \mathrm{~b}$ & $52,93 \pm 1,42 \mathrm{~b}$ & $54,99 \pm 2,18 \mathrm{~b}$ & $57,41 \pm 2,24 \mathrm{~b}$ \\
\hline \multirow{4}{*}{$\mathrm{C}^{*}$} & Cabs & $23,05 \pm 1,27 \mathrm{a}$ & $23,90 \pm 1,42 \mathrm{a}$ & $30,02 \pm 1,29 \mathrm{a}$ & $35,00 \pm 2,37 \mathrm{a}$ & $39,32 \pm 1,35 \mathrm{a}$ \\
& Controle & $23,05 \pm 1,27 \mathrm{a}$ & $23,53 \pm 1,44 \mathrm{ab}$ & $25,52 \pm 2,97 \mathrm{~b}$ & $28,69 \pm 2,09 \mathrm{~b}$ & $31,97 \pm 1,50 \mathrm{~b}$ \\
& $1 \%$ de etanol & $23,05 \pm 1,27 \mathrm{a}$ & $21,55 \pm 1,17 \mathrm{~b}$ & $22,66 \pm 1,75 \mathrm{~b}$ & $26,30 \pm 2,51 \mathrm{~b}$ & $29,11 \pm 2,49 \mathrm{c}$ \\
\hline \multirow{4}{*}{$\mathrm{H}^{*}$} & Cabs & $109,61 \pm 2,75 \mathrm{a}$ & $92,47 \pm 7,04 \mathrm{~b}$ & $78,73 \pm 7,39 \mathrm{~b}$ & $72,80 \pm 3,95 \mathrm{a}$ & $64,70 \pm 2,21 \mathrm{~b}$ \\
& Controle & $109,61 \pm 2,75 \mathrm{a}$ & $101,26 \pm 4,55 \mathrm{a}$ & $95,27 \pm 7,96 \mathrm{a}$ & $76,82 \pm 9,62 \mathrm{a}$ & $59,62 \pm 6,75 \mathrm{~b}$ \\
& $1 \%$ de etanol & $109,61 \pm 2,75 \mathrm{a}$ & $104,49 \pm 3,13 \mathrm{a}$ & $98,48 \pm 7,20 \mathrm{a}$ & $78,57 \pm 3,40 \mathrm{a}$ & $88,72 \pm 8,24 \mathrm{a}$ \\
\hline
\end{tabular}

Letras iguais na mesma Coluna não diferem significativamente entre si a $\mathrm{p} \leq 0,05$ (Teste de Tukey).

A luminosidade da polpa (Tabela 6) diminuiu com o tempo de armazenamento.Os frutos que não foram submetidos ao tratamento hidrotérmico (T1) apresentaram maiores valores para o Croma do que os que passaram por esse tratamento, essa diferença foi significativa em todos os dias de armazenamento. Em relação ao ângulo de cor, os frutos do T1 apresentaram menores valores, dados observados nos últimos dias de armazenamento, estas mangas apresentaram mais pigmentos vermelhos que as demais. Houve diferença estatística significativa no $18^{\circ}$ e $20^{\circ}$ dias de armazenamento, esses valores indicam que as mangas do controle absoluto (T1) por apresentar menor valor $\mathrm{H}^{*}$ estavam aparentemente mais maduras que os frutos dos outros tratamentos. Em estudos com goiabas Vieira et al. (2008) observaram que o tratamento hidrotérmico retardou a mudança de cor dos frutos, os mesmos autores constataram que houve aumento mais acentuado na evolução da cor durante o período de armazenamento dos frutos que não foram submetidos ao banho térmico. 
Tabela 6. Comparação dos valores médios da cor da polpa das mangas armazenadas $10^{\circ} \mathrm{C}$.

\begin{tabular}{ccccccc}
\hline Variável & Etanol & 1 Dia & 7 Dias & 14 Dias & 18 Dias & 20 Dias \\
\hline & Cabs & $78,42 \pm 0,96$ a & $77,25 \pm 1,01$ a & $76,82 \pm 1,04$ a & $72,02 \pm 1,12$ c & $68,42 \pm 0,40 \mathrm{~b}$ \\
$\mathrm{~L}^{*}$ & Controle & $78,42 \pm 0,96$ a & $76,63 \pm 1,09$ a & $77,10 \pm 0,70$ a & $75,00 \pm 0,41$ a & $71,46 \pm 0,65$ a \\
& $1 \%$ de etanol & $78,42 \pm 0,96$ a & $75,49 \pm 1,47$ a & $77,57 \pm 0,20$ a & $73,32 \pm 0,56$ b & $68,85 \pm 0,68 \mathrm{~b}$ \\
\hline & Cabs & $50,86 \pm 1,11$ a & $51,09 \pm 3,09$ c & $53,78 \pm 2,08$ a & $61,42 \pm 1,25$ a & $59,90 \pm 1,25$ a \\
$\mathrm{C}^{*}$ & Controle & $50,86 \pm 1,11$ a & $55,29 \pm 1,30$ a & $48,03 \pm 1,57$ b & $53,41 \pm 0,57$ c & $55,74 \pm 1,50 \mathrm{~b}$ \\
& $1 \%$ de etanol & $50,86 \pm 1,11$ a & $54,22 \pm 1,39$ ab & $50,62 \pm 0,56$ c & $57,36 \pm 0,78$ b & $53,48 \pm 1,26 \mathrm{c}$ \\
\hline & Cabs & $99,46 \pm 0,88$ a & $95,55 \pm 2,23$ a & $97,18 \pm 1,74$ a & $88,17 \pm 0,51$ c & $85,74 \pm 0,60 \mathrm{~b}$ \\
$\mathrm{H}^{*}$ & Controle & $99,46 \pm 0,88$ a & $94,87 \pm 2,17$ a & $97,96 \pm 0,85$ a & $92,10 \pm 0,52$ a & $87,63 \pm 1,03$ a \\
& $1 \%$ de etanol & $99,46 \pm 0,88$ a & $95,27 \pm 1,08$ a & $96,66 \pm 0,53$ a & $90,01 \pm 1,28$ b & $88,63 \pm 0,34$ a \\
\hline
\end{tabular}

Letras iguais na mesma Coluna não diferem significativamente entre si a $\mathrm{p} \leq 0,05$ (Teste de Tukey).

Os resultados da análise da aparência dos frutos encontram-se na Tabela 7. Não houve diferença estatística a 95\% de confiança pelo teste de Dunnett em todos os tratamentos em relação ao tratamento controle, portanto diante desses dados constata-se que os frutos submetidos ao tratamento hidrotérmico com $1 \%$ de etanol não apresentaram mudanças na aparência quando comparadas às amostras controle. Em estudos com uvas submetidas ao tratamento hidrotérmico associado ao etanol, Karabulut et al. (2004) e Lichter et al. (2002) não observaram alterações importantes na aparência dos frutos estudados durante todo o tempo de estocagem.

Tabela 7. Comparação das médias para o atributo aparência das mangas armazenadas a $10^{\circ} \mathrm{C}$.

\begin{tabular}{|c|c|}
\hline \multicolumn{2}{|c|}{$7^{\circ}$ dia de armazenamento a $10^{\circ} \mathrm{C}$} \\
\hline Tratamento & Aparência \\
\hline Controle Absoluto & $1,80 \pm 0,9 \mathrm{~A}$ \\
\hline Controle & $1,67 \pm 0,8 \mathrm{~A}$ \\
\hline $1 \%$ Etanol & $1,60 \pm 0,8 \mathrm{~A}$ \\
\hline DMS & 0,72 \\
\hline \multicolumn{2}{|c|}{$14^{\circ}$ dia de armazenamento a $10^{\circ} \mathrm{C}$} \\
\hline Tratamento & Aparência \\
\hline Controle Absoluto & $1,33 \pm 0,5 \mathrm{~A}$ \\
\hline Controle & $1,92 \pm 0,9 \mathrm{~A}$ \\
\hline $1 \%$ Etanol & $2,8 \pm 1,2 \mathrm{~A}$ \\
\hline DMS & 0,85 \\
\hline \multicolumn{2}{|c|}{$14^{\circ}$ dia de armazenamento a $10^{\circ} \mathrm{C}+3$ dias a $25^{\circ} \mathrm{C}$} \\
\hline Tratamento & Aparência \\
\hline Controle Absoluto & $1,80 \pm 0,7 \mathrm{~A}$ \\
\hline Controle & $2,20 \pm 0,9 \mathrm{~A}$ \\
\hline $1 \%$ Etanol & $2,40 \pm 1,1 \mathrm{~A}$ \\
\hline DMS & 0,76 \\
\hline \multicolumn{2}{|c|}{$14^{\circ}$ dia de armazenamento a $10^{\circ} \mathrm{C}+5$ dias a $25^{\circ} \mathrm{C}$} \\
\hline Tratamento & Aparência \\
\hline Controle Absoluto & $1,67 \pm 0,7 \mathrm{~A}$ \\
\hline Controle & $2,25 \pm 0,8 \mathrm{~A}$ \\
\hline $1 \%$ Etanol & $2,17 \pm 0,9 \mathrm{~A}$ \\
\hline DMS & 0,75 \\
\hline
\end{tabular}


Na Figura 1 pode-se verificar a coloração das mangas após 14 dias armazenadas a $10^{\circ} \mathrm{C} / 90 \%$ UR com transferência para $25^{\circ} \mathrm{C} / 75 \% \mathrm{UR}$ por mais 5 dias, para $\mathrm{T} 1, \mathrm{~T} 2$ e T3.

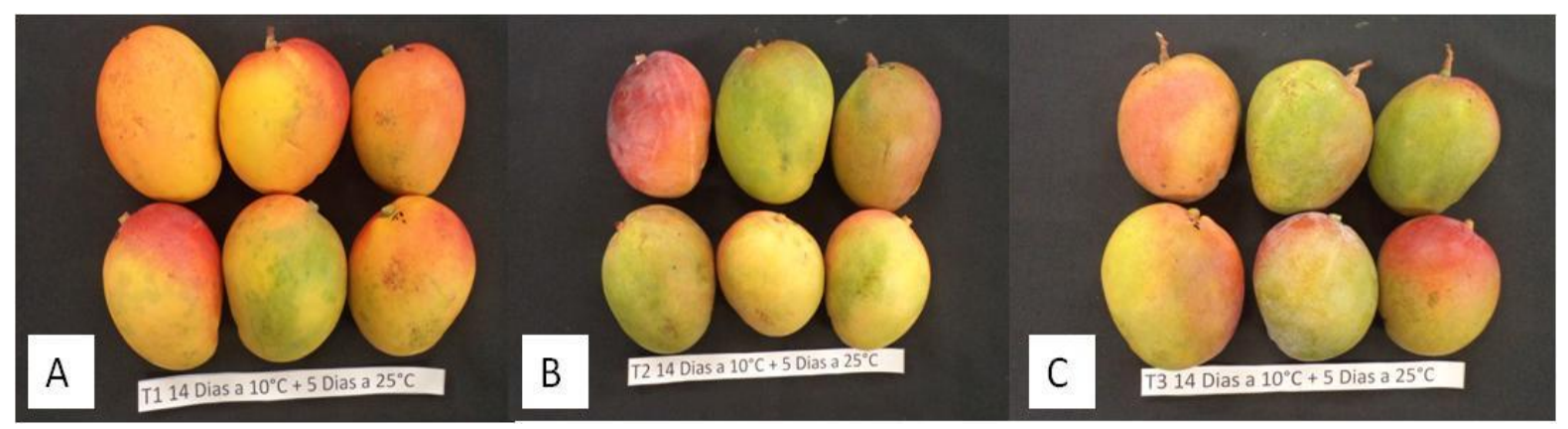

Figura 1. Mangas armazenadas por 14 dias a $10^{\circ} \mathrm{C} / 90 \%$ UR com transferência para $25^{\circ} \mathrm{C} / 75 \% \mathrm{UR}$ por mais 5 dias. $\mathrm{A}=\mathrm{T} 1, \mathrm{~B}=\mathrm{T} 2$ e $\mathrm{C}=\mathrm{T} 3$

\section{CONCLUSÕES}

As mudanças na firmeza, cor, acidez, $\mathrm{pH}$ e sólidos solúveis das mangas tratadas e não tratadas com etanol, acompanharam a evolução do amadurecimento normal do fruto.

Baseado nas alterações dos indicativos de maturação acidez, firmeza e cor pode se afirmar que a maturação foi mais rápida nas mangas apenas lavadas e armazenadas a $10^{\circ} \mathrm{C}$ (T1).

O tratamento hidrotérmico associado a $1 \%$ de etanol não danificou a aparência externa das mangas.

\section{AGRADECIMENTOS}

À FAPESP pelo apoio financeiro a essa pesquisa (Processo 10/08065-6) e ao CNPq pela bolsa de doutorado.

\section{REFERÊNCIAS BIBLIOGRÁFICAS}

ASSOCIATION OF OFFICIAL ANALYTICAL CHEMISTS (AOAC). Official methods of analysis. 16 ed. Gaithersburg-MD-USA: AOAC, 1997.

CHITARRA, M. I. F.; CHITARRA, A. B. Pós-colheita de frutas e hortaliças: fisiologia e manuseio. 2. ed. rev. e ampl. Lavras-MG: UFLA, 2005. 785p.

KARABULUT, O. A.; GABLER, F. M.; MANSOUR, M.; SMILANICK, J. L. Postharvest ethanol and hot water treatments of table grapes to control gray mold. Postharvest Biology and Technology, Elsevier, v. 34, p. 169-177, 2004.

LAURIE, S. Postharvest heat treatments of horticultural crops. Horticultural reviews, Nova Jersey-USA, v. 22. p. 91-121. 1998.

LOPES, E. B.; BRITO C. H; BATISTA, J. L.; ALBUQUERQUE I. C. Tratamento hidrotérmico no controle de larvas de ceratitis capitata em frutos de tangerina. Tecnologia \& Ciência Agropecuária, João Pessoa-PB, v. 2, n. 2, p. 23-28, 2008. 
LICHTER, A.; ZUTKHY, Y.; SONEGO, O. D.; KAPLUNOV, T.; SARIG, P.; EN-ARIE, R. Ethanol controls postharvest decay of table grapes. Postharvest Biologyand Technology, Elsevier, v. 24, p. 301-308, 2002.

MORAES, M. A. C. Métodos para avaliação sensorial dos alimentos. 6. ed. Campinas-SP: Editora da Unicamp, 1988. 93 p.

PAULL, R. E.; CHEN, N. J. Waxing and plastic wraps influence water loss from papaya fruit during storage and ripening. HortScience, Alexandria-Grécia, v. 114, p. 937-942, 1989.

PESIS, E. The role of the anaerobic metabolites, acetaldehyde and ethanol, in fruit ripening, enhancement of fruit quality and fruit deterioration. Postharvest Biology and Technology, Amsterdam, v. 37, p. 1-19, 2005

PROTRADE. Mango: Manual de exportacion: frutas tropicales y hortalizas. Eschborn: GTZ, 1992. $34 \mathrm{p}$

REZENDE, A. C. B.; CASTRO, M. F. P. M.; PORTO, E.; UCHIMA, C. A.; BENATO, E.; PENTEADO, A. L. Occurrence of Salmonella spp. in persimmon fruit (Diospyrus kaki) and growth of Salmonella Enteritidis on the peel and in the pulp of this fruit. Food Control, Elsevier, v. 20, n. 11, p. 1025-1029, 2009.

SANTOS, C. E.; BELING, R. R.; KIST, B. B.; CARVALHO, C.; REETZ, E. R.; DRUM, M. (Eds.). Anuário brasileiro de fruticultura 2014. Santa Cruz do Sul-RS: Editora Gazeta Santa Cruz, $2013 . \quad 136 \quad$ p. $\quad$ Disponível <http://www.grupogaz.com.br/tratadas/eo_edicao/4/2014/03/20140325_3d8463877/flip/>. Acesso em: 18 Fev. 2015.

SANTOS, L. O.; DURIGAN, J. F. MARTINS, R. N.; MORGADO, C. M. A. Conservação e qualidade de mangas 'palmer' submetidas a tratamento com fungicidas e hidrotérmico. Ciência e agrotecnologia, Lavras-MG, v. 34, n. 6, p. 1514-1521, nov./dez., 2010.

SILVA, D. F. P.; SALOMÃO, L. C. C.; CECON, P. R.; SIQUEIRA, D. L.; ROCHA, A. Antecipation of 'ubá' mango ripening with preharvest ethephon application. Ciência Rural, Santa Maria-RS, v. 41, n. 1, p. 63-69, 2011. Disponível em: <http://www.scielo.br/scielo.php?script=sci_arttext\&pid=S0103-84782011000100011> . Acesso em: 10 mar. 2015. doi: 10.1590/S0103-84782011000100011.

VIEIRA, S. M. J.; COUTO, S. M.; CORREAA, P. C.; SANTOS, A. E. O.; CECOM, P. R.; SILVA, D. J. P. Características físicas de goiabas (Psidium guajava L.) submetidas a tratamento hidrotérmico. Revista Brasileira de Engenharia Agrícola Ambiental, Campina Grande-RN, v. 12, n. 4, p. 408-414, 2008.

USDA-APHIS. T-102(a)- Water treatment: mango. Treatment Manual, p. 179-196. 2002. 